\title{
3D Printing of Biocompatible Supramolecular Polymers and their Composites
}

Lewis R. Hart, ${ }^{\mathrm{a}}$ Siwei Li, ${ }^{\mathrm{b}}$ Craig Sturgess, ${ }^{\mathrm{c}}$ Ricky Wildman, ${ }^{\mathrm{c}}$ Julian R. Jones ${ }^{\mathrm{b}}$ and Wayne Hayes*a

\footnotetext{
a Department of Chemistry, University of Reading, Whiteknights, Reading, RG6 6AD, U.K. e-mail: w.c.hayes@ reading.ac.uk, Tel: +44 (0)118 378 6491, Fax: +44 (0)118 3786331

${ }^{b}$ Department of Materials, Imperial College London, South Kensington Campus, London, SW7 2BP, U.K.

${ }^{c}$ Department of Chemical and Environmental Engineering, The University of Nottingham, University Park, Nottingham, NG7 2RD, U.K.
}

\begin{abstract}
A series of polymers capable of self-assembling into infinite networks via supramolecular interactions have been designed, synthesised, and characterised for use in 3D printing applications. The biocompatible polymers and their composites with silica nanoparticles were successfully utilised to deposit both simple cubic structures as well as a more complex twisted pyramidal feature. The polymers were found to be not toxic to a chondrogenic cell line, according to ISO 10993-5 and 10993-12 standard tests and the cells attached to the supramolecular polymers as demonstrated by confocal microscopy. Silica nanoparticles were then dispersed within the polymer matrix, yielding a composite material which was optimised for inkjet printing. The hybrid material showed promise in preliminary tests to facilitate the $3 \mathrm{D}$ deposition of a more complex structure.
\end{abstract}

\section{Introduction}

Additive Manufacturing (AM) and in particular 3D Printing ${ }^{1-5}$ is a rapidly developing manufacturing technology that is able to generate $3 \mathrm{D}$ objects through deposition of multiple layers of material. ${ }^{6-8}$ This process offers significant advantages over other 'traditional' subtractive manufacture techniques as a result of its ability to produce features with flexibility of design utilising a variety of different materials which, as a consequence, adds greater value to the final product. ${ }^{9}$ This value is often realised in the form of greater geometrical freedom, low to no cost personalisation and net shape manufacture. 3D printing has previously been considered primarily for prototyping but recent advances in this field mean that it is now emerging as a disruptive technology capable in large-scale production scenarios. An aspect that will facilitate this transition is the development of advanced materials that are both processable via 3D printing and possess the physical characteristics that are required in the final 
product, i.e. new 'inks' must be developed. Areas which will benefit from such precise production methods are biomedical devices and biocompatible structural components. To date, the personalised nature of 3D printing has seen the use in areas such as prosthetics and bone replacement. ${ }^{10-13}$ However, the development of high resolution, multimaterial approaches, such as ink jet printing, are opening up the applications for advanced manufacturing to the field of biodegradable or bioresorbable medical devices. Tissue regeneration strategies employ scaffolds that can act as temporary templates for tissue growth. They require complex and well-defined open pore structures to achieve enhanced cell adhesion and growth ${ }^{14-16}$ which are difficult to achieve with currently established manufacturing techniques such as foaming. In principle, 3D printing offers the capability to design, and then manufacture, complicated structures such as bone replacements.

These advantages offered by 3D inkjet printing technologies promise significant benefit for biomedical scaffolds that enable tissue regeneration and are highly desirable. ${ }^{17}$ Current ink formulations typically used for $\operatorname{coding}^{18}$ are not suitable for tissue scaffolds. Adapting existing biomaterials for inkjet deposition is not plausible as these materials do not inherently have the appropriate rheology or gelation, nor do they possess the ideal degradation rates, mechanical or bioactive properties. ${ }^{19}$ Inorganic/polymer hybrids have the potential to fulfil the criteria for an ideal scaffold, because they can comprise of co-networks of biodegradable polymers and bioactive silica. Jetting is the only potential method for the manufacture of complex 3D structures that are required for optimal scaffold architectures. Such an approach places significant restrictions on the polymer component of the ink as it must possess appropriate viscosity characteristics both in the jetting and deposition phases of the deposition process. Supramolecular polymers are ideal candidates to this end for use in hybrid jetting inks on account of their tuneable viscosities and reversible mechanical properties. $^{20}$

Supramolecular polymers have been the subject of extensive research in recent years. ${ }^{21-25}$ Developments in the design and manipulation of molecular interactions and information through recognition processes ${ }^{26-28}$ has led to the generation of a wide variety of self-assembled supramolecular arrays $^{29-33}$ in a controlled and spontaneous fashion. The stimuli-responsive properties of supramolecular polymer networks are highly desirable in value-added applications such as adhesives ${ }^{34-36}$ shape-memory materials, ${ }^{37}$ healable films ${ }^{38-45}$ and more recently in ink formulations for inkjet printing. ${ }^{20}$ Supramolecular polymer composites are also known, ${ }^{44,46,47}$ offering an improvement to the mechanical properties when compared to the polymer alone.

In this paper we describe the development of a series of biocompatible supramolecular polymer systems which can be deposited successfully in three dimensions via inkjet printing processes (Scheme 1) to form simple self-supporting structures. Hydrogen bonding motifs were appended to a biodegradable polymer, yielding supramolecular polymer networks. In a proof of concept study, these 
materials were tested for biocompatibility and also deposited using a piezoelectric inkjet printer to yield 3D structures. Upon the successful printing of simple features, the polymers were reformulating with silica nanoparticles to form hybrid solutions which were then deposited to yield more complex 3D structures.

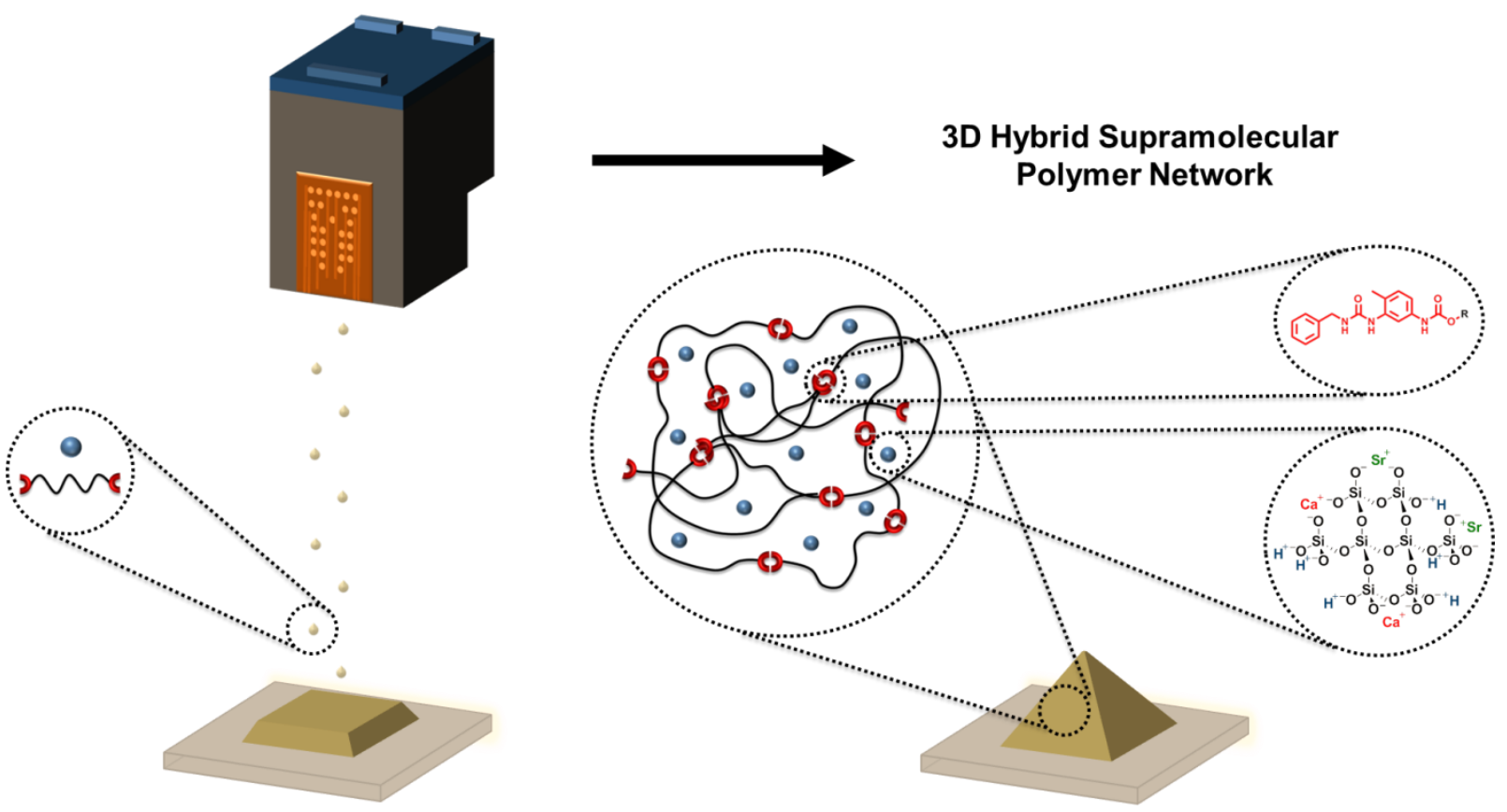

Scheme 1. Concept of 3D printing supramolecular polymer hybrids, showing silica nanoparticles (blue) and supramolecular polymers (red/black) which are deposited simultaneously to form a 3D network structure. Inserts show molecular structure of silica nanoparticles (blue) and hydrogen bonding end-group (red)

\section{Results and Discussion}

We have previously reported ${ }^{34,36,48}$ the generation of self-assembling polyurethanes whereby a hydroxyl terminated polyol was capped with a diisocyanate before conversion to a hydrogen bonding motif to facilitate assembly of the infinite supramolecular networks. By modifying this approach, biocompatible polymers which are also able to self-assemble could be used in 3D printing applications. To develop a biodegradable polymer architecture with desirable solubility characteristics, poly(caprolactone) diol $1\left(\mathrm{M}_{\mathrm{n}}=2000 \mathrm{Da}\right)$ was selected to form the polymer midblocks owing to its known degradation by acid or enzymatic hydrolysis. ${ }^{49,50}$ To gain a balance between the tensile strength elasticity and solubility of the polymer, 2,4-toluene diisocyanate $(2,4$ TDI) 2 was selected to endcap the pre-polymer 3 (Scheme 2). A series of hydrogen bonding and/or $\pi$ stacking end-groups (4a-d) were appended to the pre-polymer $\mathbf{3}$ to assess their solubility characteristics and suitability as supramolecular polymers (5a-d) in 3D printing (see Supporting Information, Figures S1-8). Amino-morpholine 4a derived end-groups are known to form selfassembled polymer networks through weak hydrogen bonds, ${ }^{36}$ whilst a strongly hydrogen bonding 
residue, namely ureidopyrimidinone $\mathrm{e}^{24,51-56}$ (UPy) 4b was selected in an attempt form more mechanically robust materials on account of the high dimerization constant of this assembly motif. Benzylamine 5c terminated polymers were expected to form hydrogen bonded and $\pi$-stacked networks. Finally, by altering the electronics of the aromatics, a motif $\mathbf{5 d}$ which was expected to strong charge-transfer interactions was selected for installation at the polymer termini. The supramolecular polymers (5a-d) were all synthesised successfully in good yields (>70 \%). ${ }^{1} \mathrm{H}$ NMR spectroscopy confirmed the formation of urethane linkages between poly(caprolactone) diol $\mathbf{1}$ and 2,4-TDI 2 through the appearance of a multiplet resonance at $4.2 \mathrm{ppm}$. Probe resonances from the end-group, namely the methylene protons adjacent to the newly formed urea/urethane or methyl groups within the moieties, were used to assess the formation of the desired polymer structure. Endgroup analysis revealed that the pre-polymer $\mathbf{3}$ had been end-capped with minimal chain extension (less than $10 \%$ ) of the pre-polymer. Infrared spectroscopy analysis also demonstrated the loss of the isocyanate signals at $c a .2360 \mathrm{~cm}^{-1}$ in all of the polymers, demonstrating complete consumption of the isocyanate in the final polymer, and thus end-capping of the pre-polymer.

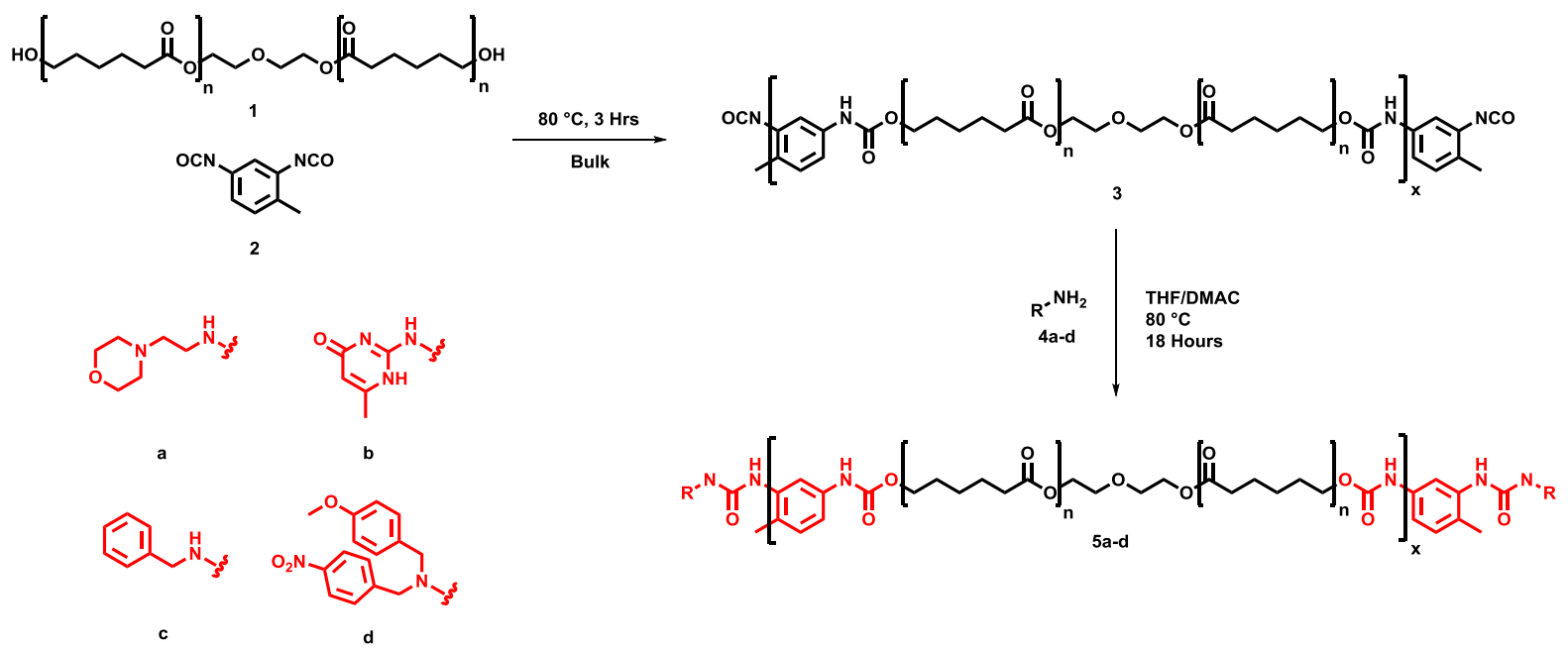

Scheme 2. Synthesis of poly(caprolactone) derived supramolecular polymers (5a-d).

With the supramolecular polymers in hand, dynamic solution viscosities (Figure S9) were then analysed via rheometry using a Peltier cylinder cartridge. In addition to chloroform, DMAc was selected as a co-solvent as a result of its viscosity characteristics $(0.95 \mathrm{cP})$, boiling point $\left(165^{\circ} \mathrm{C}\right)$ and lower vapour pressure $(0.30 \mathrm{kPa})$ to give a balance between solubilising capability, viscosity and evaporation rate of the inkjet fluid. Polymers 5a-d (150 mM, ca. $\left.400 \mathrm{mg} \mathrm{mL}^{-1}\right)$ were dissolved in a blend of DMAc/Chloroform (2:1, v/v), resulting in viscosities ranging between 10-25 cP over a range of sheer rates. This parameter range was in keeping with the required viscosity of $10 \mathrm{cP}$ or greater for optimal printing on a piezoelectric drop-on-demand inkjet printer. ${ }^{57}$ The UPy terminated polymer $\mathbf{5 b}$ which revealed sheer-thinning characteristics with increased sheer. This may be attributed to the supramolecular network being more easily disrupted by higher shear forces as the viscosity of the polymer appears to converge with other polymers within the series at higher shear rates. 
To assess the drop forming properties of the supramolecular polymers $\mathbf{5 a}-\mathbf{b}$, solutions $(150 \mathrm{mM}$ in DMAc/chloroform, 2:1 (v/v)) were filtered (5 $\mu \mathrm{m}$ microfiltration membrane) and the drop shape analysed (Figure S10). All polymers were observed to have an average surface tensions of between 26 and $29 \mathrm{mN} \mathrm{m}^{-1}$ when measured over a series of 10 drops, which fall close to the ideal value of $28 \mathrm{mN}$ $\mathrm{m}^{-1}$ and above. ${ }^{58,59}$

To develop the waveform which was used to deposit the polymer solution, a test printer rig (Figure S11) was utilised to mimic the piezoelectric drop-on-demand inkjet printer. An inkjet cartridge was filled with a solution of 5a and the waveform altered to explored jet-ability. The waveform can be split into 5 distinct sections. In the initial stage the nozzle is at rest, then, by applying a zero voltage in stage two, the ink is drawn into the nozzle. In the third and fourth stage, the ink is dispelled from the nozzle by applying the maximum voltage and subsequently decreasing the voltage. In the final stage, the nozzle was returned to rest and so the waveform cycle can begin again. The drop break-up and ligament formation may be altered by the amount of voltage applied in the fourth and fifth stage. From this, two candidate waveforms were developed (Figure S12) which were observed to jet material onto a piece of white card and also visually as a stream of droplets. Both were developed at ambient temperature using an operating voltage of $27 \mathrm{~V}$, a frequency of $1 \mathrm{MHz}$, and a meniscus vacuum of $0.0 \mathrm{~Pa}$.

Drop formation was further analysed using the Dimatix drop watcher (Figure S13). All of the polymers were analysed using waveform ' $a$ ' and ' $b$ ' as described in (Figure S12) at ambient temperature using an operating voltage of $27 \mathrm{~V}$, a frequency of $1 \mathrm{MHz}$, and a meniscus vacuum of 25.0 Pa. Both waveforms proved effective when printing on the piezoelectric drop-on-demand printer, resulting in consistent drop break-up when deposited under analogue conditions as developed on the test-rig printer. Stable drops of the amino morpholine terminated polyurethane 5a were able to be deposited with a ligament attached initially. At a distance of $c a .100 \mu \mathrm{m}$, the ligament was seen to coalesce with the leading drop to form a single droplet. Drop break-up was observed to be diminished when using waveform ' $b$ '. The drop forming characteristics of the UPy end-capped polymer $\mathbf{5 b}$ was good when using waveform 'a', but was observed to diminish over time, possible as a result of nozzle wetting. The benzylamine terminated polymer 5c was observed to form more stable drops when compared to 5a, with only small ligaments forming when using waveform 'a', however, the polymer was not printable using waveform ' $b$ '. Finally, the droplet formation of polymer 5d was improved greatly when using waveform ' $b$ ', forming single drops without ligaments after travelling a distance of $120 \mu \mathrm{m}$.

To assess the suitability of the supramolecular polymers (5a-d) in forming three-dimensional structures, a $5 \times 5 \mathrm{~mm}$ square of the individual polymers were deposited onto a glass slide and each 
sample built up to 300 layers with drying time between layers added to aid the process (taking a total of 4 hours for each feature). To facilitate drying, a 10 second pause was inserted between printing each layer (Figure S14). All materials were deposited, producing features which were less than $1 \mathrm{~mm}$ in height in the z-axis. The aminomorpholine terminated polymer 5a showed signs of unstable drop break-up as demonstrated by poor image resolution and blurring of the feature. Deposition of the UPy end-capped polymer $\mathbf{5 b}$ resulted in a colourless printed image which had moderately good resolution, but appeared to not be as thick as the other deposited samples. Deposition of the benzyl terminated polymer 5c afforded the best resolution and definition of the series of polymer whilst the nitro/methoxy dibenzylamine terminated polymer $\mathbf{5 d}$ demonstrated improved jet stability and resulted in a well-defined feature, although not as sharp as 5c. From these preliminary results, the benzyl terminated polymer 5c was selected for further investigations. A feature which was 700 layers in thickness was deposited resulting in a height of $c a .1 .5 \mathrm{~mm}$. Figure 1 shows polymer $\mathbf{5 c}$ after 700 layers, which demonstrated an enhancement in the height of the feature, although a small amount of resolution is observed to be lost owing to the material beginning to 'dome' as increasing layers of solvated material is deposited.

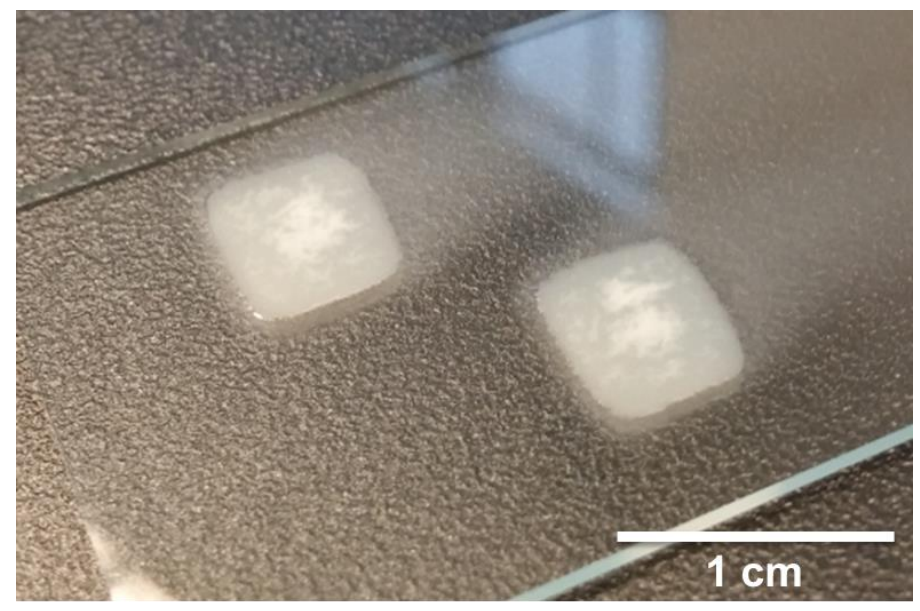

Figure 1. Images of 2 features printed from polymer 5c. Each feature represents 700 layers and was allowed to dry in ambient conditions over night before the material fully solidified.

Films cast of polymers 5a, 5c and $\mathbf{5 d}$ were also assessed for cytotoxicity. MTT assays confirmed that all polymer compositions analysed did not induce cytotoxic effects (Figure 2), performed in accordance to ISO 10993-5 and 10993-12. It is noted in ISO 10993, for a material to be deemed biocompatible, the viability of cells cultured in its $100 \%$ liquid extracts should not fall below $70 \%$ in comparison to blank and negative (non-toxic) control. 
$5 a$

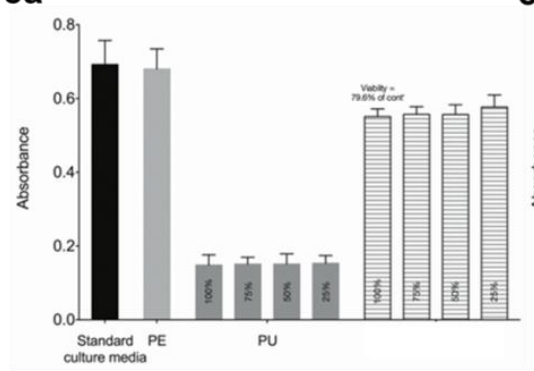

5c

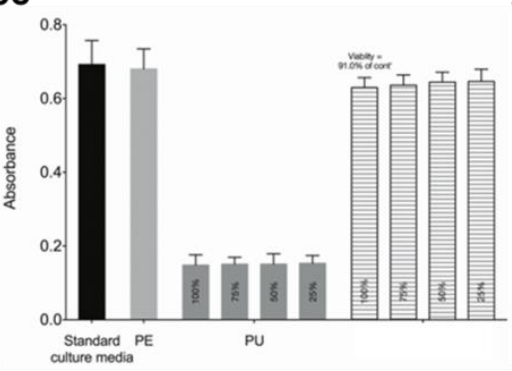

5d

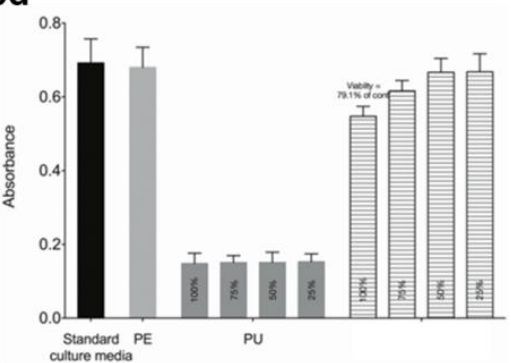

Figure 2. Cytotoxicity testing of the polymers 5a, c and $\mathbf{d}$ by MTT assays of the dissolution products of the polymers.

Cell attachment on the new materials was examined by immunohistochemistry and confocal microscopy. As shown in Figure 3, the expression of the Vimentin and Actin cytoskeletal protein was evident in ATDC5 cells seeded on polymer membranes following 24 hours of culture. Upon examination of cytoskeletal proteins and cellular morphology, ATDC5 cell attachment on the polymers 5a, 5c and $\mathbf{5 d}$ was not affected by the addition of weak hydrogen bonding/ $\pi-\pi$ stacking motifs to the biocompatible poly(caprolactone).
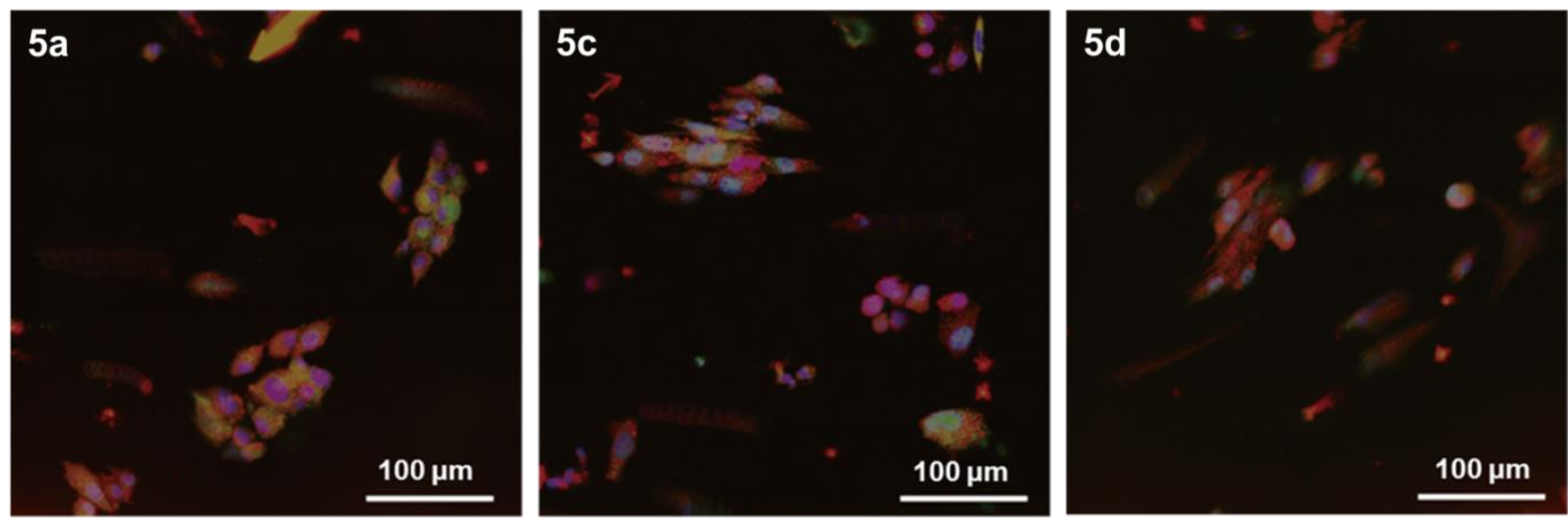

Figure 3. Confocal microscopy demonstrating cell attachment to the supramolecular polymers $\mathbf{5 a}$, c and $\mathbf{d}$ using Vimentin and Actin staining.

From this proof of concept it can be concluded that inkjet deposition of supramolecular polymers to form three-dimensional structures is possible. To further improve these mechanical characteristics of these materials and for potential in the generation of biocompatible bone scaffolds, hybrids were developed containing silica particles $(7 \mathrm{~nm})$ which were incorporated into the inkjet printing formulations. The particles (Bindzil ${ }^{\circledR}$ CC 301) were supplied as a colloidal dispersion in water. To deploy these spherical silica particles in the polymer ink formulations, a solvent exchange with DMAc was undertaken. The silica dispersion (39\% silica w/v) was mixed with an equal volume of DMAc and heated under reflux using a Dean-Stark apparatus for 5 hours to remove the water content. The solvent exchange resulted in a solution as opposed to dispersion, indicating the particles were completely solubilised by DMAc. Mixing this solution with chloroform resulted in instantaneous 
precipitation of the silica particles. For this reason, the polymer hybrids were deposited from DMAc alone. The benzylamine terminated polymer $\mathbf{5 c}$ was selected to conduct polymer hybrid printing studies. Blending solutions of 5c (150 mM) in DMAc with the silica particles $(5 \%, 7.5 \%$, or $10 \%$, $\mathrm{w} / \mathrm{v})$ in DMAc resulted in turbid liquids. The dispersion was filtered through a $5 \mu \mathrm{m}$ microfiltration membrane, which also resulted in a turbid solution which was suitable for printing. To assess the presence of any agglomerates, microscopy of the solutions was undertaken (Figure S15). Particle loadings of 5\% and $7.5 \%$ demonstrated good dispersion in solution as well as particle sizes of agglomerates less than $5 \mu \mathrm{m}$. From this data it was clear that it should be possible to deposit the polymer hybrid as the nozzle width in the printer cartridge is $30 \mu \mathrm{m}$.

The viscosity of the hybrid solutions of $\mathbf{5 c}$ was analysed (Figure 4). A viscosity increase was observed for all hybrid solutions when compared to the polymer solution alone, with some shear thinning observed at low sheer rates. Loadings of $5 \%$ silica $(15.1 \mathrm{cP})$ demonstrated an increase in viscosity of $50 \%$ when compared to the viscosity of $\mathbf{5 c}$ alone, whilst a $7.5 \%$ loading of silica exhibited a viscosity increase of $60 \%(16.2 \mathrm{cP})$. The dispersion containing $10 \%$ silica particles (w/v) initially revealed dramatic sheer thinning, before yielding a viscosity of $c a .22 \mathrm{cP}$ over a range of shear rates.

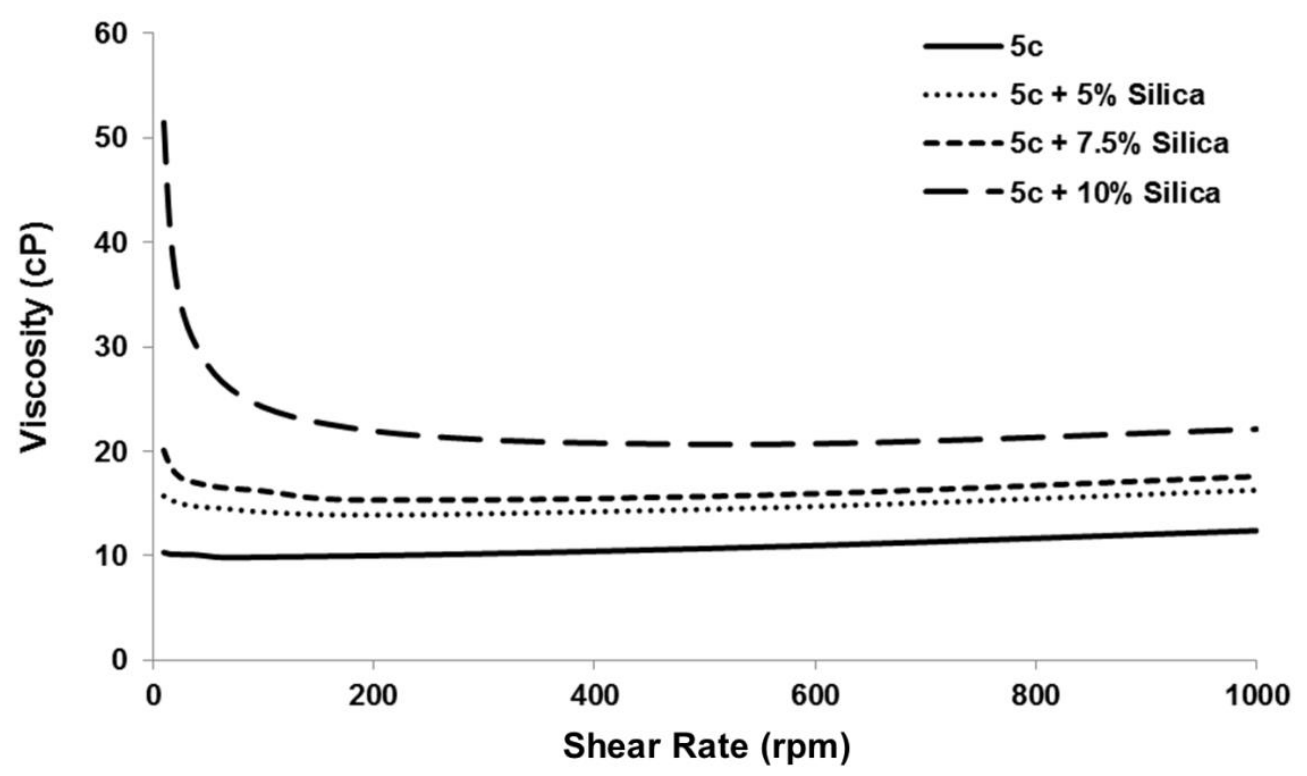

Figure 4. Viscosities of the benzyl terminated supramolecular polymer (5c) hybrids at different concentrations of silica particles.

The hybrid solutions were then transferred into cartridges for analysis for the drop formation. The break-up of the drops of hybrid inks were observed to be less stable than that of the pure polymer formulations (Figure S16). Whilst jet stability was seen to be good, occasionally drops were ejected in a non-linear fashion, diverging from the vertical plane. Increasing the loading of hybrid material exacerbated this effect, with the $10 \%$ silica formulation proving to be very unstable. For this reason, the $5 \%$ and $7.5 \%$ hybrids were used in the printing studies. Hybrid materials were deposited onto 
clean glass slides. Again, a $5 \times 5 \mathrm{~mm}$ square was printed (Figure 5) of the individual polymers and each sample built up to 300 layers, allowing time between layers to aid the polymer drying. The height of the features was noted to be greater $(c a .1 .5 \mathrm{~mm})$ than that of the polymer alone. Furthermore, the features produced were observed to have columns build up within the overall structure, owing to the resolution of the printer which deposits the drops in rosters. By creating these columns, it should be noted that hybrid materials are able to form defined structures which are self-supporting, a desirable property for creating more intricate structures for regenerative medicine and more widely, biomedical applications.

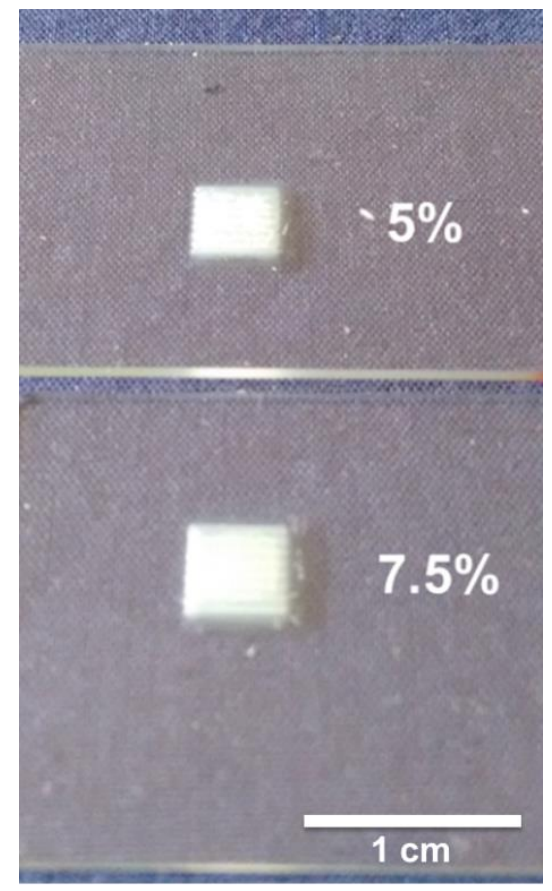

Figure 5. Image of the printed samples of $\mathbf{5 c}$ containing 5\% and 7.5\% silica deposited onto clean glass slides. Each feature is 300 layers in thickness.

Finally, the dispersion of silica in the printed samples was analysed by environmental scanning electron microscopy (ESEM) and also energy-dispersive X-ray spectroscopy (EDX). SEM images of the printed hybrids and also the pure polymer formulation (Figure S17a) revealed the morphology of the features formed. The surface of the deposited materials appears to be homogeneous, with individual drops evident. It is also evident that cracks are formed during the drying process but it must be emphasised that these ink formulations are rudimentary in nature. The edges of the features formed are not well-defined, instead presenting a rounded shape. The definition is, however, significantly improved, with more precise square edges observed as increased silica loadings were utilised. The columns formed during the printing of these hybrids were also clearly evident in the micrographs, perhaps more clearly in the 5\% silica loading formulation. Further magnification (Figure S17b) revealed small volumes of polymer deposited, possibly by individual drops, which shows a tight packing in all materials deposited. Feature sizes are in the order of 10-20 $\mu \mathrm{m}$. 
SEM/EDX spectroscopy analysis (Figure 6) of the printed features of the benzyl terminated polymer 5c gave insight to the distribution of silica particles in the hybrid materials. Increasing loadings of silica were observed in the printed hybrid features when compared to the pure polymer formulation, although no clear difference is noted between the 5\% and $7.5 \%$ loadings.
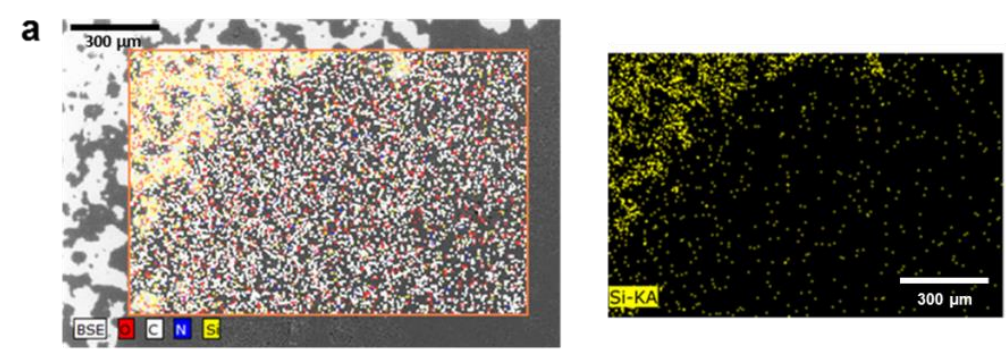

b
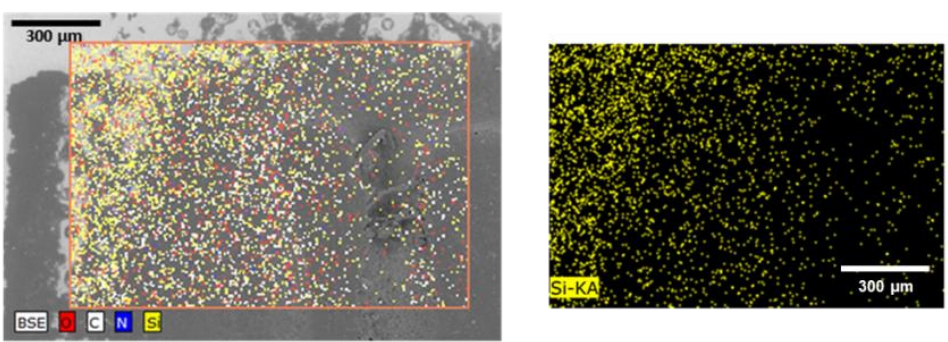

C
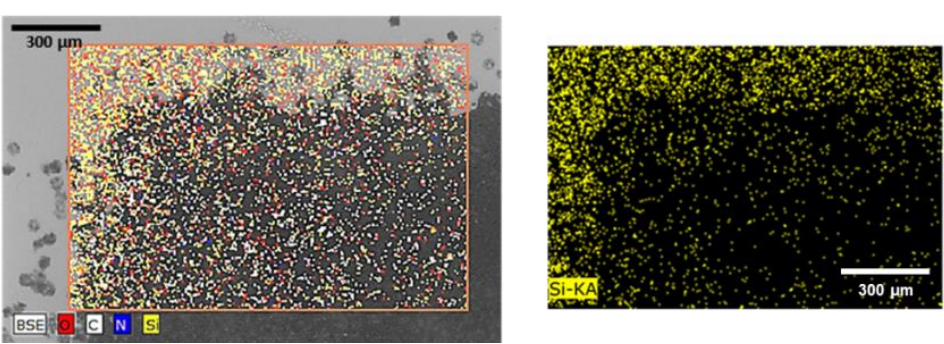

Figure 6. EDX spectroscopy analysis of the printed features of a: the benzyl terminated polymer $\mathbf{5 c}$, b: a hybrid between $\mathbf{5 c}$ and $5 \%$ silica, and c: the hybrid between $\mathbf{5 c}$ and $7.5 \%$ silica.

To further examine the use of hybrid materials in inkjet deposited 3D structures, a more intricate feature was printed. A twisted pyramid design (Figure 7a) was envisaged which would be selfsupporting whilst demonstrating structural complexity. The CAD design was split into 20 crosssections and each layer deposited 100 times to build up the twisting pyramid. The pyramid design $(6 \times$ $6 \mathrm{~mm}$ base) was successfully printed with a feature height of $3 \mathrm{~mm}$ once complete (Figure $7 \mathrm{~b}$ and $7 \mathrm{c}$ ) before allowing to fully dry at room temperature, yielding a solid structure. Examination of the feature revealed a small amount of splattering from the inkjet, whilst a twist could be observed as per the CAD model. 

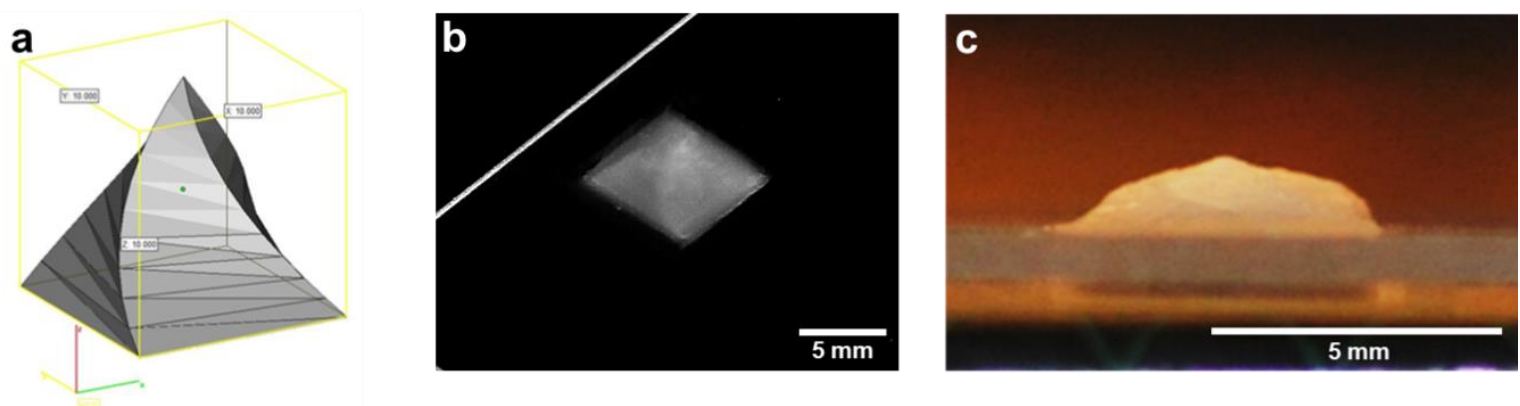

Figure 7. a: CAD design of the twisted pyramid, b: photograph of the pyramid showing the twisted design and c: profile view of the printed material.

\section{Conclusions}

Novel biocompatible supramolecular polymers have been designed, synthesised and tested for application in a 3D inkjet printer to generate features which represent the first steps towards supramolecular polymer based hybrid scaffolds for regenerative medicine. A biodegradable poly(caprolactone) diol $\left[\mathrm{M}_{\mathrm{n}}=2000 \mathrm{Da}\right]$ was appended with a series of hydrogen bonding moieties yielding supramolecular polymer with desirable solubility characteristics. Initial studies developed two waveforms and printing parameters which facilitated the deposition of all polymers. A benzyl terminated polymer $(\mathbf{5 c})$ was observed to produce the best printed features with respect to image resolution and was thus selected to take forward in hybrid material studies. Silica particles were loaded into the polymer formulation at a number of different weight to volume ratios and formulations of $\mathbf{5 c}$ with $5 \%$ silica (w/v) or $7.5 \% \%(\mathrm{w} / \mathrm{v})$ were successfully deposited as simple patterns. Analysis of these printed features allowed further understanding of the properties generated by the inclusion of hybrid particles in the printed features. A self-supporting twisted pyramid was also printed to demonstrate that more detailed constructs could be deposited via the reported method. The biocompatibility of the polymers was also tested; cytotoxicity studies revealed that the polymers were non-toxic in accordance with ISO 10993-5 and 10993-12. Cell attachment was also examined, revealing cell attachment was not affected by the addition of hydrogen bonding motifs to the biocompatible poly(caprolactone), as confirmed by confocal microscopy. This proof of concept study has demonstrated promising results towards the use of polymer hybrid materials which may be 3D printed to form biomedical scaffolds for regenerative medicine and there is excellent scope for supramolecular materials and their composites in 3D printing of complex structures.

\section{Experimental}


Reagents and solvents were purchased from Sigma Aldrich and were used without further purification, with the exception of dichloromethane which was dried by distillation from calcium hydride under argon. Cell culture reagents were purchased from Invitrogen UK or Sigma-Aldrich UK unless specified otherwise. Proton NMR (400 MHz) and ${ }^{13} \mathrm{C}$ NMR (100 MHz) spectra were obtained on a Bruker Nanobay 400 spectrometer using $\mathrm{CDCl}_{3}$ or $\mathrm{CDCl}_{3} /$ trifluoroacetic acid $(9: 1 \mathrm{v} / \mathrm{v})$ as solvent, with TMS as internal standard. Infrared (IR) spectroscopic analysis was carried out using a Perkin Elmer 100 FT-IR instrument with diamond-ATR sampling accessory and samples either as solids or oils. Gel Permeation chromatography (GPC) data were collected using an Agilent Technologies 1260 Infinity Series chromatograph. Samples were dissolved in THF which was also used as the eluent, with poly(styrene) standards for calibration. Differential scanning calorimetry (DSC) was carried out using a TA Instruments Q2000 calorimeter. Samples for DSC were heated to $110{ }^{\circ} \mathrm{C}$ to remove residual solvent, cooled to $-90{ }^{\circ} \mathrm{C}$, and then re-scanned from -90 to $250{ }^{\circ} \mathrm{C}$. Environmental Scanning Electron Microscope (ESEM) images of polymer films were recorded with a FEI Quanta FEG 600 ESEM equipped with a hot stage for polymer films. Initial printing was carried out on a custom designed printer (Mr Craig Sturgess, University of Nottingham) whilst 3D printing was undertaken using a Fujifilm Dimatix Materials Printer DMP-2800 series using 11600 series Dimatix materials cartridges, the same printer was also used to collect data using the drop watcher feature. Viscosities of the printed samples were collected using A Malvern Kinexus Pro+, using a Peltier cylinder cartridge. Drop shape analysis was undertaken using a Kruss DSA 100. Environmental Scanning Electron Microscope (ESEM) images printed images were collected using a Hitachi Analytical Benchtop SEM TM3030 plus with EDX system. In vitro cell culture studied were conducted using a chondrogenic cell line ATDC5 (ATCC, UK) which was culture expanded in monolayer cultures in basal DMEM supplemented with 5\% (v/v) FCS (foetal calf serum), $100 \mathrm{unit} / \mathrm{ml}$ penicillin, $100 \mu \mathrm{g} / \mathrm{ml}$ streptomycin and $1 \times$ ITS liquid supplement $(10 \mu \mathrm{g} / \mathrm{ml}$ insulin, $5.5 \mu \mathrm{g} / \mathrm{ml}$ transferrin and $5 \mathrm{ng} / \mathrm{ml}$ selenite premix). Cultures were maintained in humidified atmosphere at $37^{\circ} \mathrm{C}$, $5 \% \mathrm{CO}_{2}$ and $21 \% \mathrm{O}_{2}$. Cells were passaged upon confluence using $500 \mu \mathrm{g} / \mathrm{ml}$ trypsin-EDTA (ethylene diamine tetra-acetic acid). Potential in vitro cytotoxicity effects of the supramolecular polymers was analysed on ATDC5 cells which were assessed in accordance to ISO 10993-5 and ISO 10993-12. Liquid extracts of the samples $\left(3 \mathrm{~cm}^{2} / \mathrm{ml}\right.$ in DMEM at $\left.37^{\circ} \mathrm{C}\right)$ over a 72 hour period were prepared. Medical grade polyethylene (PE) was used as negative control (non-cytotoxic) and polyurethane (PU) containing $0.1 \%(\mathrm{w} / \mathrm{w})$ zinc diethyldithiocarbamate (ZDEC) was used as positive control (provide reproducible cytotoxic response). The liquid extracts were filter sterilised and, dilution series (25\%, $50 \%, 75 \%$ and $100 \%$ ) were prepared prior to use in cell viability assays. Cell viability was assessed by a calorimetric cell metabolic activity assay based on the conversion of 3-(4,5-dimethylthiazol-2-yl)-2,5-diphenyltetrazolium bromide (MTT) into formazan. ATDC5 cells were seeded on 96-well plates at $1 \times 10^{4}$ cells per well and left to grow in basal DMEM for 24 hours until a sub-confluent monolayer was formed. The culture media was removed and, cells were then 
incubated with fresh basal DMEM, the liquid extracts of polymer films or controls (100 $\mu 1 /$ well) for further 24 hours. The culture media was removed and MTT solution $(1 \mathrm{mg} / \mathrm{ml}$ in serum-free DMEM) was added $(50 \mu 1 /$ well). Following an incubation period of 2 hours, the MTT solution was removed and each well was filled with $100 \mu 1$ isopropanol and shaken briefly to dissolve the formazan derivatives. The optical density was measured spectrophotometrically at $570 \mathrm{~nm}$ using a microplate reader (SpectraMax M5). Cell attachment studies were carried out on polymer films (approximately $5 \times 5 \times 1 \mathrm{~mm}^{3}$ ) which were prepared and sterilised with $70 \%$ ethanol for 1 minute. Following washing with PBS, each sample was placed in serum-free $\alpha$-MEM for 30 minutes prior to cell seeding. Monolayer expanded ATDC5 cells were harvested and suspended in basal $\alpha$-MEM at a concentration $1 \times 10^{6}$ cells $/ \mathrm{ml} .10 \mu \mathrm{l}$ of cell suspension was seeded onto each polymer membrane and, incubated in humidified atmosphere at $37^{\circ} \mathrm{C}, 5 \% \mathrm{CO}_{2}$ and $21 \% \mathrm{O}_{2}$ for 2 hours. Each cell-seeded membrane was then submerged in fresh basal DMEM and cultured for further 24 hours. Cell-seeded membranes were fixed with 4\% paraformaldehyde (PFA) and used for immunohistochemical analysis of cell attachment. Following permeabilisation with buffered 0.5\% Triton X-100 in PBS (300 mM sucrose, $50 \mathrm{mM} \mathrm{NaCl}, 3 \mathrm{mM} \mathrm{MgCl} 2,20 \mathrm{mM}$ Hepes and $\mathrm{pH}$ 7.2) and blocking with $10 \mathrm{mg} / \mathrm{ml} \mathrm{BSA}$ in PBS, samples were incubated with anti-Vimentin antisera (1:500 dilution in $10 \mathrm{mg} / \mathrm{ml} \mathrm{BSA}$ in PBS, rabbit polyclonal, IgG, Abcam, Cambridge, UK) at $4^{\circ} \mathrm{C}$ for 1 hour. This was followed by hour-long incubation with Alexa Fluor ${ }^{\circledR}$ 488-conjugated secondary antibody. Negative controls (omission of the primary antisera) were performed in all immunohistochemistry procedures. No staining was observed in the samples used as negative controls. F-actin was labelled using CytoPainter F-actin staining kit (Abcam, Cambridge, UK) following the manufacture's instruction. Briefly, Alexa Fluor ${ }^{\circledR}$ 568conjugated phalloidin (1:1000 dilution in labelling buffer) was added simultaneously with the secondary antibody during the incubation period. All samples were counter-stained with DAPI $(0.1 \mu \mathrm{g} / \mathrm{ml}$ in PBS). The samples were imaged under confocal microscopy (Leica SP5 MP laser scanning confocal microscope and software, Leica Microsystems, Wetzlar, Germany).

\section{Acknowledgements}

We thank EPSRC and University of Nottingham for an 'Exploring the Science behind AM \& 3DP' post-doctoral fellowship grant for LRH. SL and JRJ also thank EPSRC (EP/I020861/1) for additional funds. Spectroscopic and thermal data were acquired using instrumentation in the Chemical Analysis Facility (CAF) at the University of Reading. The authors are grateful to the Electron Microscopy Laboratory (EMLab) at the University of Reading for providing access to the ESEM microscope facility. We would also like to thank, Mark East, Mark Hardy, Yinfeng He, Benjamin Paul and Joe White, of the University of Nottingham for constructive assistance with the 3D printing studies. We thank AkzoNobel for supplying a sample of Bindzil ${ }^{\circledR}$ CC 301. The technical advice on nanoparticle 
selection provided by Professor Steven P. Armes from the University of Sheffield is also acknowledged.

\section{References}

(1) Wendel, B.; Rietzel, D.; Kühnlein, F.; Feulner, R.; Hülder, G.; Schmachtenberg, E. Additive Processing of Polymers. Macromol. Mater. Eng. 2008, 293, 799-809.

(2) Chia, H. N.; Wu, B. M. Recent Advances in 3D printing of Biomaterials. J. Biol. Eng. 2015, 9, 4.

(3) Tseng, P.; Murray, C.; Kim, D.; Di Carlo, D. Research Highlights: Printing the Future of Microfabrication. Lab Chip 2014, 14, 1491-1495.

(4) Shirazi, S. F. S.; Gharehkhani, S.; Mehrali, M.; Yarmand, H.; Metselaar, H. S. C.; Adib Kadri, N.; Osman, N. A. A. A Review on Powder-based Additive Manufacturing for Tissue Engineering: Selective Laser Sintering and Inkjet 3D Printing. Sci. Technol. Adv. Mater. 2015, 16,033502 .

(5) Murphy, S. V; Atala, A. 3D Bioprinting of Tissues and Organs. Nat. Biotechnol. 2014, 32, 773-785.

(6) Lipson, H.; Kurman, M. Fabricated: The New World of 3D Printing; John Wiley \& Sons, Inc., 2013.

(7) Farahani, R. D.; Chizari, K.; Therriault, D. Three-dimensional Printing of Freeform Helical Microstructures: A Review. Nanoscale 2014, 6, 10470-10485.

(8) Zhang, M.; Vora, A.; Han, W.; Wojtecki, R. J.; Maune, H.; Le, A. B. A.; Thompson, L. E.; McClelland, G. M.; Ribet, F.; Engler, A. C.; Nelson, A. Dual-Responsive Hydrogels for Direct-write 3D Printing. Macromolecules 2015, 48, 6482-6488.

(9) Hague, R.; Campbell, I.; Dickens, P. Implications on Design of Rapid Manufacturing. Proc. Inst. Mech. Eng. Part C J. Mech. Eng. Sci. 2003, 217, 25-30.

(10) Jiang, C.-P.; Huang, J.-R.; Hsieh, M.-F. Fabrication of Synthesized PCL-PEG-PCL Tissue Engineering Scaffolds Using an Air Pressure-aided Deposition System. Rapid Prototyp. J. 2011, 17, 288-297.

(11) Butscher, A.; Bohner, M.; Hofmann, S.; Gauckler, L.; Müller, R. Structural and Material Approaches to Bone Tissue Engineering in Powder-based Three-dimensional Printing. Acta Biomater. 2011, 7, 907-920.

(12) Eshraghi, S.; Das, S. Mechanical and Microstructural Properties of Polycaprolactone Scaffolds with One-dimensional, Two-dimensional, and Three-dimensional Orthogonally Oriented Porous Architectures Produced by Selective Laser Sintering. Acta Biomater. 2010, 6, 24672476.

(13) Mannoor, M. S.; Jiang, Z.; James, T.; Kong, Y. L.; Malatesta, K. a.; Soboyejo, W. O.; Verma, N.; Gracias, D. H.; McAlpine, M. C. 3D Printed Bionic Ears. Nano Lett. 2013, 13, 2634-2639. 
(14) Bobyn, J. D.; Stackpool, G. J.; Hacking, S. A.; Tanzer, M.; Krygier, J. J. Characteristics of Bone Ingrowth and Interface Mechanics of a New Porous Tantalum Biomaterial. J. Bone Jt. Surgery. 1999, 81, 907-914.

(15) Hacking, S. A.; Bobyn, J. D.; Toh, K. K.; Tanzer, M.; Krygier, J. J. Fibrous Tissue Ingrowth and Attachment to Porous Tantalum. J. Biomed. Mater. Res. 2000, 52, 631-638.

(16) Bobyn, J. D.; Pilliar, R. M.; Binnington, A. G.; Szivek, J. A. The Effect of Proximally and Fully Porous-coated Canine Hip Stem Design on Bone Modeling. J. Orthop. Res. 1987, 5, 393-408.

(17) Kemp, P. History of Regenerative Medicine: Looking Backwards to Move Forwards. Regen. Med. 2006, 1, 653-669.

(18) Magdassi, S. The Chemistry of Inkjet Inks; S. Magdassi, Ed.; World Scientific Publishing Co. Pte. Ltd.: Singapore, 2010.

(19) Jones, J. R. Review of Bioactive Glass: From Hench to Hybrids. Acta Biomater. 2013, 9, $4457-4486$.

(20) Hart, L. R.; Harries, J. L.; Clifton, A.; Colquhoun, H. M.; Hayes, W. Inkjet Composition. Patent App. No: WO 2014111722 A1, 2014.

(21) Brunsveld, L.; Folmer, B. J. B.; Meijer, E. W.; Sijbesma, R. P. Supramolecular Polymers. Chem. Rev. 2001, 101, 4071-4098.

(22) Lehn, J.-M. Supramolecular Polymer Chemistry - Scope and Perspectives. Polym. Int. 2002, $51,825-839$.

(23) Ciferri, A. Supramolecular Polymerizations. Macromol. Rapid Commun. 2002, 23, 511-529.

(24) Bosman, A. W.; Sijbesma, R. P.; Meijer, E. W. Supramolecular Polymers at Work. Mater. Today 2004, 7, 34-39.

(25) Huang, F.; Scherman, O. A. Supramolecular Polymers. Chem. Soc. Rev. 2012, 41, 5879-5880.

(26) Colquhoun, H. M.; Zhu, Z. Recognition of Polyimide Sequence Information by a Molecular Tweezer. Angew. Chem., Int. Ed. 2004, 43, 5040-5045.

(27) Colquhoun, H. M.; Zhu, Z.; Cardin, C. J.; Gan, Y. Principles of Sequence-recognition in Aromatic Polyimides. Chem. Commun. 2004, 2650-2652.

(28) Colquhoun, H. M.; Zhu, Z.; Cardin, C. J.; Gan, Y.; Drew, M. G. B. Sterically Controlled Recognition of Macromolecular Sequence Information by Molecular Tweezers. J. Am. Chem. Soc. 2007, 129, 16163-16174.

(29) Iverson, B. L.; Lokey, R. S. Synthetic Molecules That Fold into a Pleated Secondary Structure in Solution. Nature 1995, 375, 303-305.

(30) Murr, M. M.; Harting, M. T.; Guelev, V.; Ren, J.; Chaires, J. B.; Iverson, B. L. An Octakisintercalating Molecule. Bioorg. Med. Chem. 2001, 9, 1141-1148.

(31) Nguyen, J. Q.; Iverson, B. L. An Amphiphilic Folding Molecule that Undergoes an Irreversible Conformational Change. J. Am. Chem. Soc. 1999, 121, 2639-2640. 
(32) Lehn, J.-M. Supramolecular Chemistry - Scope and Perspectives Molecules - Supermolecules and Molecular Devices. Angew. Chem., Int. Ed. 1988, 27, 89-112.

(33) Lehn, J.-M. Perspectives in Supramolecular Chemistry - From Molecular Recognition towards Molecular Information Processing and Self-Organization. Angew. Chem., Int. Ed. 1990, 29, 1304-1319.

(34) Woodward, P.; Clarke, A.; Greenland, B. W.; Hermida Merino, D.; Yates, L.; Slark, A. T.; Miravet, J. F.; Hayes, W. Facile Bisurethane Supramolecular Polymers Containing Flexible Alicyclic Receptor Units. Soft Matter 2009, 5, 2000-2010.

(35) Hermida Merino, D.; Slark, A. T.; Colquhoun, H. M.; Hayes, W.; Hamley, I. W. Thermoresponsive microphase separated supramolecular polyurethanes. Polym. Chem. 2010, 1, 12631271.

(36) Woodward, P. J.; Hermida Merino, D.; Greenland, B. W.; Hamley, I. W.; Light, Z.; Slark, A. T.; Hayes, W. Hydrogen Bonded Supramolecular Elastomers: Correlating Hydrogen Bonding Strength with Morphology and Rheology. Macromolecules 2010, 43, 2512-2517.

(37) Li, J.; Viveros, J. A.; Wrue, M. H.; Anthamatten, M. Shape-Memory Effects in Polymer Networks Containing Reversibly Associating Side-groups. Adv. Mater. 2007, 19, 2851-2855.

(38) Greenland, B. W.; Burattini, S.; Hayes, W.; Colquhoun, H. M. Tetrahedron 2008, 64, 83468354.

(39) Burattini, S.; Colquhoun, H. M.; Fox, J. D.; Friedmann, D.; Greenland, B. W.; Harris, P. J. F.; Hayes, W.; Mackay, M. E.; Rowan, S. J. Design, Synthesis and Computational Modelling of Aromatic Tweezer-molecules as Models for Chain-folding Polymer Blends. Chem. Commun. 2009, 6717-6719.

(40) Burattini, S.; Colquhoun, H. M.; Greenland, B. W.; Hayes, W. A Novel Self-healing Supramolecular Polymer System. Faraday Discuss. 2009, 143, 251-264.

(41) Burattini, S.; Greenland, B. W.; Chappell, D.; Colquhoun, H. M.; Hayes, W. Healable Polymeric Materials: A Tutorial Review. Chem. Soc. Rev. 2010, 39, 1973-1985.

(42) Burattini, S.; Greenland, B. W.; Hermida Merino, D.; Weng, W.; Seppala, J.; Colquhoun, H. M.; Hayes, W.; Mackay, M. E.; Hamley, I. W.; Rowan, S. J. A Healable Supramolecular Polymer Blend Based on Aromatic $\pi-\pi$ Stacking and Hydrogen-bonding Interactions. J. Am. Chem. Soc. 2010, 132, 12051-12058.

(43) Burattini, S.; Greenland, B. W.; Hayes, W.; Mackay, M. E.; Rowan, S. J.; Colquhoun, H. M. A Supramolecular Polymer Based on Tweezer-Type $\pi-\pi$ Stacking Interactions: Molecular Design for Healability and Enhanced Toughness. Chem. Mater. 2011, 23, 6-8.

(44) Fox, J.; Wie, J. J.; Greenland, B. W.; Burattini, S.; Hayes, W.; Colquhoun, H. M.; Mackay, M. E.; Rowan, S. J. High-strength, Healable, Supramolecular Polymer Nanocomposites. J. Am. Chem. Soc. 2012, 134, 5362-5368.

(45) Hart, L. R.; Harries, J. L.; Greenland, B. W.; Colquhoun, H. M.; Hayes, W. Healable Supramolecular Polymers. Polym. Chem. 2013, 4, 4860.

(46) Tee, B. C.-K.; Wang, C.; Allen, R.; Bao, Z. An Electrically and Mechanically Self-healing Composite with Pressure- and Flexion-sensitive Properties for Electronic Skin Applications. Nat. Nanotechnol. 2012, 7, 825-832. 
(47) Vaiyapuri, R.; Greenland, B. W.; Rowan, S. J.; Colquhoun, H. M.; Elliott, J. M.; Hayes, W. Thermoresponsive Supramolecular Polymer Network Comprising Pyrene-Functionalized Gold Nanoparticles and a Chain-Folding Polydiimide. Macromolecules 2012, 45, 5567-5574.

(48) Feula, A.; Pethybridge, A.; Siviour, C. R.; Buckley, C. P.; Hamley, I. W.; Hayes, W. A Thermoreversible Supramolecular Polyurethane with Excellent Healing Ability at $45{ }^{\circ} \mathrm{C}$. Macromolecules 2015, 48, 6132-6141.

(49) Yasin, M.; Tighe, B. Polymers for Biodegradable Medical Devices. J. Biomaterials 1992, 13, 9-16.

(50) Siparsky, G. L.; Voorhees, K. J.; Miao, F. Hydrolysis of Polylactic Acid (PLA) and Polycaprolactone (PCL) in Aqueous Acetonitrile Solutions: Autocatalysis. J. Environ. Polym. Degrad. 1998, 6, 31-41.

(51) Sijbesma, R. P.; Beijer, F. H.; Brunsveld, L.; Folmer, B. J.; Hirschberg, J. H.; Lange, R. F.; Lowe, J. K.; Meijer, E. W. Reversible Polymers Formed from Self-complementary Monomers using Quadruple Hydrogen Bonding. Science 1997, 278, 1601-1604.

(52) Beijer, F. H.; Sijbesma, R. P.; Kooijman, H.; Spek, A. L.; Meijer, E. W. Strong Dimerization of Ureidopyrimidones via Quadruple Hydrogen Bonding. J. Am. Chem. Soc. 1998, 120, 67616769.

(53) Hirschberg, J. H. K. K.; Beijer, F. H.; Aert, H. A. Van; Magusin, P. C. M. M.; Sijbesma, R. P.; Meijer, E. W. Supramolecular Polymers from Linear Telechelic Siloxanes with QuadrupleHydrogen-Bonded Units. Macromolecules 1999, 32, 2696-2705.

(54) Hirschberg, J. H.; Brunsveld, L.; Ramzi, A; Vekemans, J. A; Sijbesma, R. P.; Meijer, E. W. Helical Self-assembled Polymers from Cooperative Stacking of Hydrogen-bonded Pairs. Nature 2000, 407, 167-170.

(55) Feldman, K. E.; Kade, M. J.; Meijer, E. W.; Hawker, C. J.; Kramer, E. J. Phase Behavior of Complementary Multiply Hydrogen Bonded End-Functional Polymer Blends. Macromolecules 2010, 43, 5121-5127.

(56) Aida, T.; Meijer, E. W.; Stupp, S. I. Functional Supramolecular Polymers. Science 2012, 335, 813-817.

(57) Brunahl, J. Piezoelectric Shear Mode Drop-on-demand Inkjet Actuator. Sens. Actuators, A 2002, 101, 371-382.

(58) Yun, Y. H.; Kim, J. D.; Lee, B. K.; Cho, Y. W.; Lee, H. Y. Polymer Inkjet Printing: Construction of Three-dimensional Structures at Micro-scale by Repeated Lamination. Macromol. Res. 2009, 17, 197-202.

(59) Fujifilm Dimatix Inc. Materials Printer. Jettable Fluid Formulation Guidelines, 2013. 